\title{
TIPOLOGÍA DE SISTEMAS DE PRODUCCIÓN EN LA ZONA RURAL DEL MUNICIPIO DE IBAGUÉ, COLOMBIA ${ }^{1}$
}

\author{
Cristina Rocha-Rodríguez ${ }^{2}$, Jairo Mora-Delgado ${ }^{2}$, Jenny Catalina Romero-Vargas ${ }^{3}$
}

\begin{abstract}
RESUMEN
Tipología de sistemas de producción en la zona rural del municipio de Ibagué, Colombia. El objetivo de este estudio fue realizar una tipificación de las fincas de la zona rural de Ibagué, Colombia. Se aplicaron 55 encuestas entre marzo y abril del 2013, en cinco corregimientos. La encuesta abarcó aspectos humanos, sociales, físicos, financieros y naturales. La tipificación evidenció tres grupos de fincas: el grupo 1 poseía una importante proporción del suelo bajo pasturas $(3,71 \pm 5,88 \mathrm{ha})$ y eran sistemas típicos de economía campesina, en los cuales se combinaban actividades agrícolas y pecuarias. El grupo 2 estuvo conformado básicamente por fincas dedicadas al cultivo del café; su superficie era pequeña $(3,15 \pm 2,31$ ha). En el grupo 3 predominaron las pasturas; fueron fincas con características poco comunes, particularmente por su gran extensión $(52,43 \pm 111,51$ ha). Predominó la diversidad productiva, tanto agrícola como pecuaria, y también la heterogeneidad social típica de las sociedades campesinas.
\end{abstract}

Palabras clave: comunidad campesina, usos de la tierra, ganadería, agricultura.

\begin{abstract}
Typology of production systems in the rural area of Ibague, Colombia. The aim of this study was to perform a classification of the farms in the rural area of Ibague, Colombia. Between March and April 2013, 55 surveys were applied in five districts. The survey covered human, social, physical, financial, and natural aspects. The classification showed three types of farms: group 1 had a significant proportion of soil under pastures $(3.71 \pm 5.88 \mathrm{ha})$ and these were typical systems of peasant economy, which combine agricultural and livestock activities. Group 2 was basically made up of farms dedicated to grow coffee; its surface was small (3.15 $\pm 2,31 \mathrm{ha})$. In group 3 , farms with land use under pastures predominated; these farms had atypical characteristics, particularly by its size $(52.43 \pm 111.51$ ha). Both agricultural and livestock productive diversity predominated, as well as the typical social heterogeneity of peasant societies.
\end{abstract}

Keywords: peasant community, land uses, livestock, agriculture.

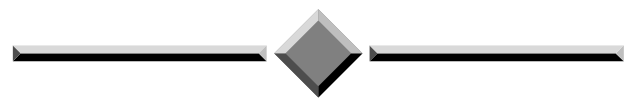

\footnotetext{
1 Recibido: 19 de mayo, 2015. Aceptado: 13 de agosto, 2015. Este estudio forma parte del trabajo final de graduación de la Maestría en Ciencias Pecuarias del primer autor, Tecnología y conocimiento local de técnicas alimentarias en una tipología de los sistemas de producción pecuarios según zonas de vida en el área rural de Ibagué. Universidad del Tolima, Ibagué, Colombia.

2 Universidad del Tolima, Grupo de Investigación Sistemas Agroforestales Pecuarios, Altos de Santa Elena, Ibagué, Colombia. girafita@ hotmail.com,jrmora@ut.edu.co (autor para correspondencia).

3 Universidad del Tolima, Profesional de apoyo Oficina Central de Proyección Social, Altos de Santa Elena, Ibagué, Colombia. jcromerov@ ut.edu.co
} 


\section{INTRODUCCIÓN}

El municipio de Ibagué, ubicado en el departamento de Tolima, cuenta con una importante despensa agrícola que llena en gran medida las necesidades alimentarias de la población que habita la ciudad capital de dicho departamento. Los diversos rangos altitudinales y por tanto, la conformación geográfica de la zona hacen que en este territorio exista una amplia oferta de sistemas de producción agropecuarios, con un gran potencial para el abastecimiento alimentario, situación que contrasta con la presencia de sectores marginales (Alcaldía de Ibagué, 2010). A pesar de estar en permanente contacto con la zona urbana, lo cual constituye una supuesta irradiación de los beneficios de la ciudad, sus habitantes aún conservan prácticas económicas y expresiones culturales típicos de las sociedades rurales.

Existe un importante número de estudios de la zona rural del municipio de Ibagué (Gobernación del Tolima, 2011; Rodríguez y Mora, 2015), en cuyo territorio se tiene una gran diversidad productiva, pero estos no caracterizan la diversidad de los sistemas de producción y organización social. Por consiguiente, la complejidad de los mismos no es abordada como un objeto de análisis, lo que constituiría una base para el diseño de políticas diferenciadas o para la asistencia técnica con base en el concepto dominios de recomendación (Berdegué y Larraín, 1988). Así, la realización de una tipología brinda las herramientas para tal fin.

El término "tipología" puede usarse para designar el ejercicio de elaboración de tipos, diseñado para ayudar a analizar una realidad compleja y el orden de los objetos que, a pesar de ser diferentes, son de un mismo tipo, p. ej. una finca (Landais, 1998). Por otra parte, puede usarse el concepto para designar el sistema de tipos resultante de este proceso (la tipología granja de una determinada región). La tipificación busca agrupar a productores con características de manejo, producción y técnicas similares; algunos productores están ubicados en zonas geográficas delimitadas (Berdegué y Larrain, 1992).

La tipología de productores puede basarse en estudios realizados previamente y en la información con la que cuentan los entes territoriales, buscando tener un primer acercamiento con los lugares objeto de estudio. Luego se hace necesario hacer un trabajo de campo donde se tenga contacto directo con los productores, para obtener información que se analice buscando llegar a conclusiones que permitan mejorar los sistemas productivos de la región (García y Calle, 1998).

El objetivo de este estudio fue realizar una tipificación de las fincas de la zona rural de Ibagué, Colombia.

\section{MATERIALES Y MÉTODOS}

\section{Zona de estudio}

El presente estudio se realizó en la zona rural del municipio de Ibagué, en el departamento del Tolima, que cuenta con una amplia zona rural muy diversificada, tanto en arreglos productivos, como en condiciones topográficas, que van desde laderas superiores al $80 \%$ en la parte alta de la cuenca del río Combeima, hasta tierras planas en la terraza de Ibagué. El municipio está ubicado en las coordenadas: $\mathrm{N} 4^{\circ}$ $15^{\prime}$ a N $4^{\circ} 40^{\prime}$ y W 750 $00^{\prime}$ a 75³0 $30^{\prime}$ (Vanegas, 2002), y sus franjas altitudinales varían entre 800 y $5215 \mathrm{msnm}$, sin embargo, es hasta los $2600 \mathrm{msnm}$ que registra la actividad agropecuaria de mayor importancia.

Ibagué se encuentra dividido en diecisiete corregimientos y 140 veredas, de los cuales, para fines de este estudio, se abarcaron cinco corregimientos por considerarlos los de mayor actividad antrópica y concentración predial (Salado, San Juan de la China, San Bernardo, Totumo y Carmen de Bulira), situados al oriente del municipio (Figura 1). La población rural es cercana a los 30000 habitantes, y representa el 6\% del total del municipio.

Se consultaron fuentes secundarias y se hizo un acercamiento previo a la comunidad con los líderes locales. Posteriormente, una búsqueda en el Instituto Geográfico Agustín Codazzi (IGAC) y de material cartográfico, permitió obtener una aproximación del número total de predios rurales del municipio de Ibagué y la distribución de los usos agropecuarios del suelo en la zona. Una análisis de la distribución predial de la cuenca del río Combeima, permitió tener una idea de las características de la estructura agraria de la zona de estudio, así se estimó un total de 1589 predios debidamente legalizados ante el IGAC, de los cuales el 98\% tienen extensión menor o igual a 100 ha (Guio et al., 2014).

El contacto con la Unidad Municipal de Asistencia Técnica (UMATA) del municipio de Ibagué, permitió obtener la lista de contactos de los corregidores y los presidentes de junta de cada una de las veredas, 


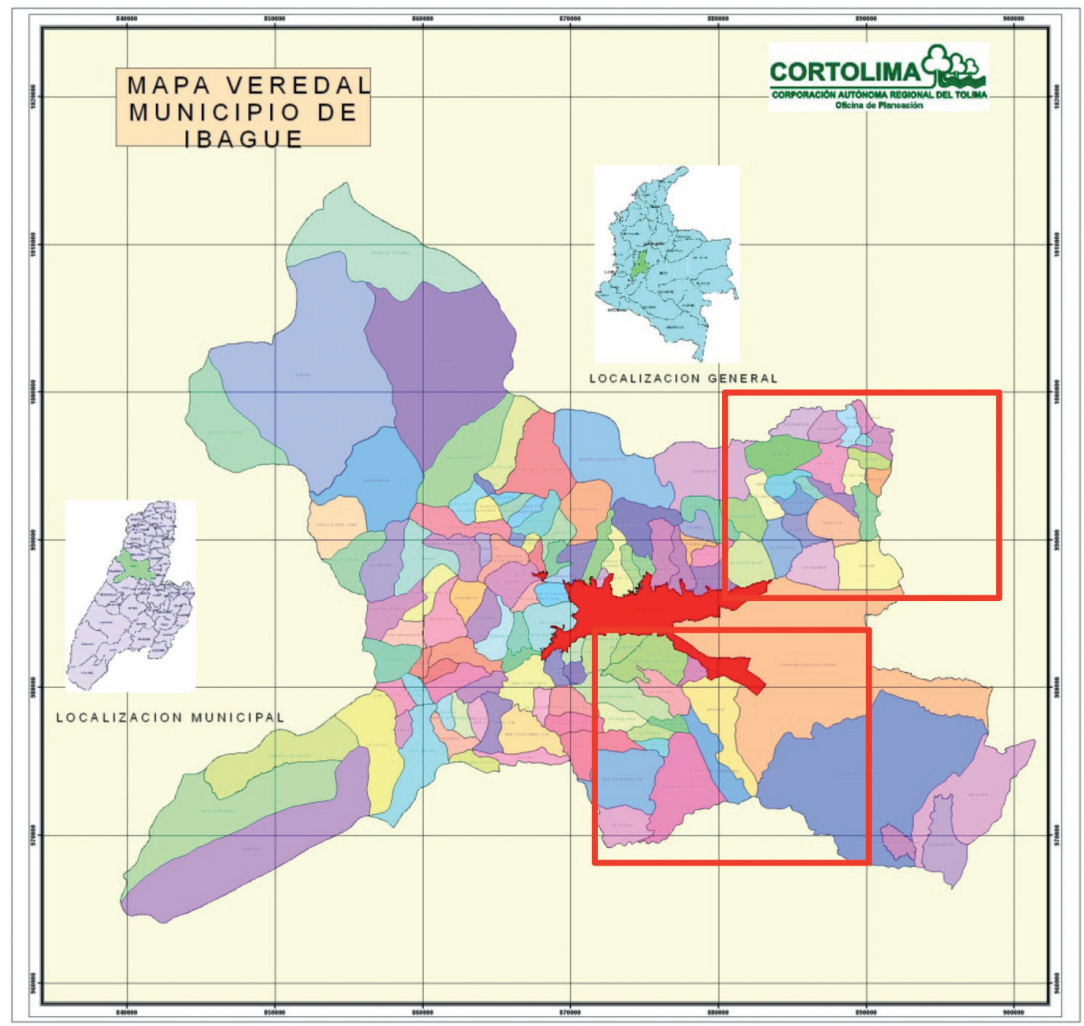

Figura 1. Mapa del municipio de Ibagué (Colombia) donde se resaltan los corregimientos donde se tipificaron los sistemas de producción en la zona rural. Fuente: Alcaldía de Ibagué (2010).

Figure 1. Map of Ibague (Colombia), districts where production systems in the rural were tripled are highlighted. Source: Alcaldía de Ibagué (2010).

quienes fueron fundamentales para los ingresos a la zona y la triangulación de información.

\section{Muestreo}

Se realizaron reuniones con productores y caminatas por las localidades, para establecer contactos directos con presidentes de organizaciones locales, quienes facilitaron el acercamiento y sensibilización de las familias a la presencia de los investigadores, prerrequisito para posteriormente recabar la información mediante entrevistas y cuestionarios. Así, se determinó cuáles de estas localidades tenían interés para el estudio; este ejercicio exploratorio constituyó la base para un muestreo intencional, también denominado "por conveniencia".

En el muestreo intencional se seleccionaron los objetos que se estimaba eran representativos o típicos de la población. En tal selección es importante el criterio del experto o el investigador para elegir las unidades, que se estima, pueden facilitar la información necesaria (Hernández-Sampieri et al., 2006; Murray y Stephens, 2009; Bolaños, 2012). Normalmente, en este muestreo se establecen criterios de conveniencia para la aplicabilidad de la muestra, los cuales para este estudio fueron: 1) predios preferiblemente de economía campesina; 2) que mostraran sistemas mixtos, con componente agrícola y pecuario; y 3 ) anuencia de la familia a participar en el proyecto.

Una vez obtenido el mapa productivo general de la zona de estudio, se realizó el muestreo de 55 fincas distribuidas en tres franjas altitudinales, siguiendo la aproximación de Stiles (1998): a) tierras bajas, por debajo de $1000 \mathrm{msnm}$; b) elevaciones medias, entre 1000-2000 msnm; c) elevaciones superiores a 
2000-2600 msnm. Teniendo en cuenta que la mayor concentración predial se encontraba dentro de las dos primeras franjas (800 - $2000 \mathrm{msnm}$ ) se decidió aplicar un mayor número de encuestas en esta área.

\section{Recolección de la información}

Se diseñó un cuestionario estructurado con preguntas sobre aspectos demográficos, sociales, físicos, financieros y ambientales, cuya información se tabuló en una hoja de Excel en la cual se seleccionaron las variables mostradas en el Cuadro 1. Con base en estas variables se hizo un análisis estadístico multivariado, que permitió hacer un agrupamiento de las fincas.

\section{Metodología de tipificación}

El análisis de la información se realizó siguiendo la metodología propuesta por Bermúdez et

Cuadro 1. Notaciones matemáticas de las variables utilizadas para la tipificación de fincas en la zona rural del municipio de Ibagué, Colombia. 2013.

Table 1. Mathematical notation of the variables used for typing farms in the rural area of the municipality of Ibagué, Colombia. 2013.

\begin{tabular}{lll}
\hline Nombre de la variable & Notación matemática & Observaciones \\
\hline Integrantes & $I E=F(1) \sum_{i=1}^{K} n X i($ grados $)$ & $\begin{array}{l}\text { Número de personas que conforman el hogar. Donde: n: } \\
\text { número de integrantes de la familia, Xi: i-ésima característica } \\
\text { Índice educativo }\end{array}$ \\
& $\begin{array}{l}\text { para el grado con i: } 1,2,3 \ldots \mathrm{K}: \text { características, F(1): factor de } \\
\text { corrección, preescolar: 0,2, primaria: } 0,3, \text { secundaria: } 0,5, \\
\text { técnico: } 0,7, \text { universitario: } 1,0, \text { posgrado } 1,2 .\end{array}$ \\
\hline
\end{tabular}

Percepción de las organizaciones

$\operatorname{Per}=1+\sum_{i=1}^{K} \operatorname{Or}(F)$

Índice de participación

$\operatorname{Par}=\sum_{n=i}^{K}(X i)$

Área cultivos

$A C: \sum A C$

Área pastos

$A P: \sum A P$

Artefactos

$A: \sum A r$

Capacidad instalada

$l: \sum l n$

Ingresos agrícolas

$l A: \sum \ln A g$

$l P: \sum \ln P e$

Ingresos pecuarios $\quad l P: \sum \ln P e$

$\mathrm{K}$ : mención de organizaciones presentes en la vereda, F: factor de corrección para la clasificación dada por el productor para cada una de las organizaciones presentes dada de la siguiente manera: nada importante $(0,2)$; poco importante $(0,4)$; importante $(0,6)$; muy importante $(=, 8)$; fundamental (1).

Donde Xi: valor absoluto de la i-ésima variable: número de formas de organización a las que pertenece; n: número de acciones colectivas realizadas y participación o no en actividades de capacitación.

Donde AC: áreas de la finca dedicadas a diferentes tipos de cultivos.

Donde AP: área total de la finca establecida en pasturas.

Donde Ar: la suma de los artefactos presentes en la finca, considerados como artefactos: los sistemas de riego, vehículo, bomba de espalda, bomba de agua, picadora de pasto, motosierra, generador eléctrico, despulpadora y tractor.

Donde In: es la presencia de instalaciones con fines pecuarios o agrícolas tales como: cercas vivas, cercas muertas, bodegas, invernaderos agrícolas, corrales, porquerizas, galpones y estanques piscícolas.

Donde InAg: ingresos totales provenientes de actividades agrícolas.

Donde IP: ingresos totales provenientes de actividades pecuarias. 
al. (2011), con los siguientes pasos: 1) revisión y selección de variables para el análisis de tipificación y clasificación; se identificaron aquellas variables que contribuían a la clasificación de los predios, eliminando información redundante; las variables seleccionadas fueron a discreción de los investigadores. 2) Análisis de componentes principales (CP); se llevó a cabo utilizando diez variables de 55 fincas, con el fin de reducir el número de variables con pérdida mínima de información. 3) Análisis de conglomerados o análisis de cluster (AC), el cual permitió implementar distintos procesos para agrupar las fincas con base en un conjunto de valores de varias variables (Balzarini et al., 2008). 4) Análisis discriminante canónico (ADC), este permitió confirmar el conjunto de variables de mayor peso en la discriminación para la formación de los grupos o clúster.

\section{Análisis estadístico}

Las diferencias entre grupos de las variables analizadas, fueron determinadas mediante diferencia de medias con una prueba de $T$ de student $(\mathrm{p} \leq 0,05)$, mediante el software Infostat (Di Rienzo et al., 2008). A partir de esta clasificación se hicieron estudios de caso y se analizaron las variables más relevantes para caracterizar las fincas centrómeros de cada grupo.

\section{RESULTADOS Y DISCUSIÓN}

\section{Análisis de componentes principales (CP)}

Los autovalores o eigenvalues, mostraron que el primer componente, con un valor $\lambda=2,49$, explicó el $25 \%$ de la variación, el segundo componente explicó el $21 \%(\lambda=2,12)$, el tercero el $14 \%(\lambda=1,40)$ y el cuarto, con un valor $\lambda=1,05$ que explica el $10 \%$ de la variación. Estos cuatro primeros componentes explicaron el $71 \%$ acumulado de la variación del total de la muestra. Es notorio un decrecimiento a partir del cuarto valor; es decir, los cuatro primeros explicaron la mayoría de los componentes. Esto permite la reducción de las variables de las diez originales a las tres siguientes: primer componente (agrícola): área total de cultivos, ingresos agrícolas, índice educativo; segundo componente (instalaciones y desarrollo pecuario): artefactos, capacidad instalada e ingresos pecuarios; y tercer componente (social): percepción de organizaciones, integrantes del hogar y pastos.
El primer componente se encontró explicado por el área total de cultivos, los ingresos agrícolas y el índice educativo, con coeficientes de 0,44, 0,54 y 0,47 , respectivamente. El segundo componente se encontró por ingresos pecuarios, capacidad instalada y artefactos, con coeficientes de 0,52, 0,53 y 0,47, respectivamente. El tercer componente presentó los coeficientes mayores para percepción a organizaciones con un valor de 0,73 , pastos con un valor de $0,59 \mathrm{y}$ por último, integrantes del hogar con un valor de 0,21.

\section{Análisis de conglomerados (AC)}

El agrupamiento obtenido en el análisis de conglomerados se muestra en la Figura 2.

\section{Análisis discriminante canónico (AD)}

Buscando determinar cuáles fueron las variables que más relevancia tuvieron en la caracterización de los tres grupos, se realizó un análisis discrimínate canónico, con lo cual se pudo establecer que la primera función explicó un 83,83\% de la variación, considerando dentro de esta total de cultivos, pastos e ingresos pecuarios como las tres variables de mayor peso. La segunda función explicó el 16,17\%, tuvo como variables más importantes el índice educativo y el índice de participación.

El dendrograma (Figura 2) mostró tres grupos con características diferenciadoras entre sí, dos de ellos fueron de un tamaño similar mientras que el tercero tuvo una cantidad de fincas menor; es decir, las características de dichas fincas no son comunes dentro de las fincas encuestadas. Mora et al. (2011) reportaron un dendograma similar, pero la variabilidad en el tamaño de estos fue menor que la encontrada en el presente estudio, lo cual sugiere la existencia de tres grupos de fincas consolidados, con características distintivas entre sí. En el estudio realizado hubo un grupo (G3) con pocas unidades de análisis, comparado con los otros dos. Dicho grupo de fincas presentó características de mayor desarrollo y alta inversión con respecto a los otros dos grupos.

\section{Características sociales}

El número de integrantes del grupo familiar en la muestra analizada, considerando los tres grupos, varió entre una y siete personas con un promedio 


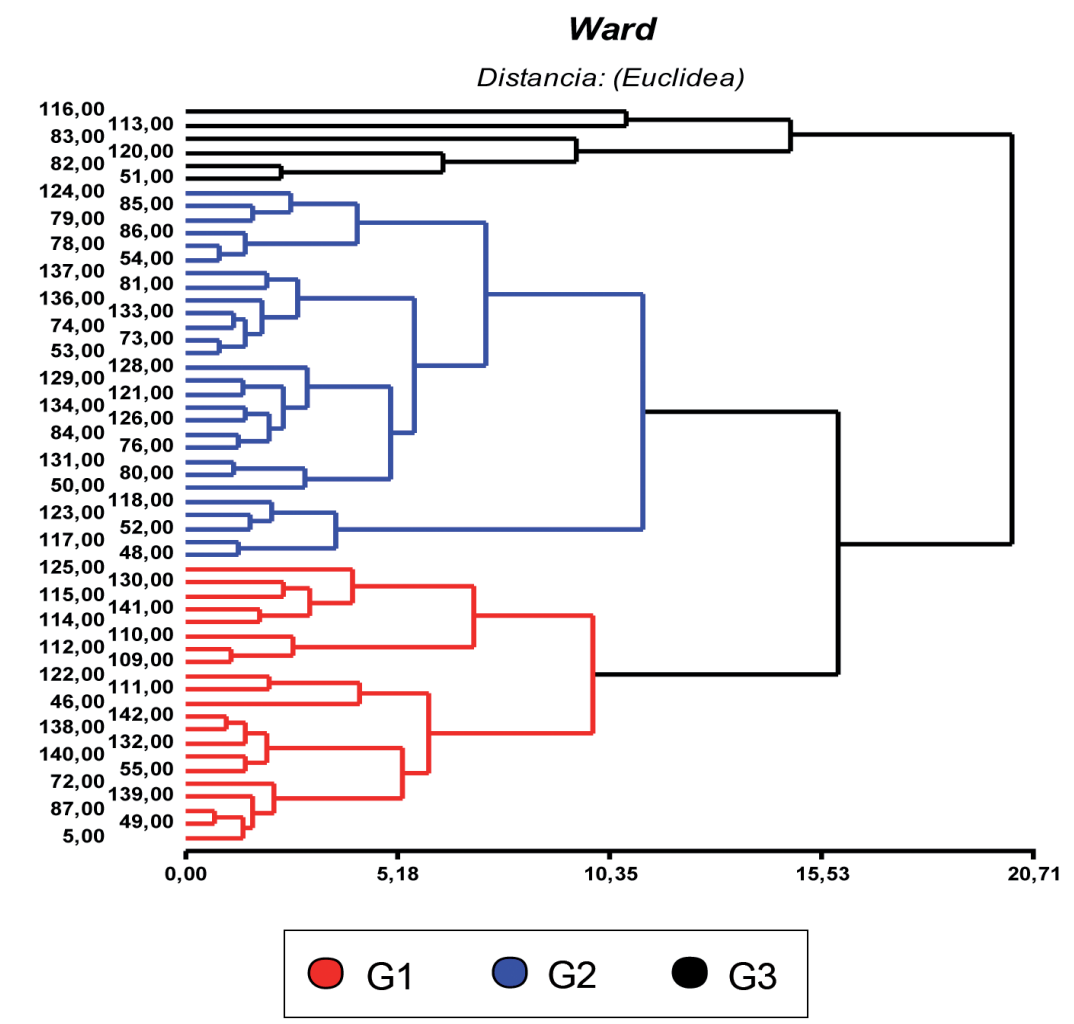

Figura 2. Dendograma del análisis de conglomerados, según la técnica de Ward, para 63 fincas de cinco corregimientos de Ibagué, Colombia. 2013. G1: grupo 1; G2: grupo 2; G3: Grupo 3.

Figure 2. Dendrogram of cluster analysis according to Ward technique for 63 farms in five districts of Ibague, Colombia. 2013. G1: group 1; G2: group 2; G3: group 3 .

de $3,6 \pm 1,5$, lo cual indicó la existencia de una población con hogares conformados por un número de integrantes reducido, integrados por parejas jóvenes, así confirmado en el análisis de la distribución etárea. La mayor parte de las familias eran biparentales y nucleadas (Cuadro 2).

La media encontrada en la muestra analizada fue menor a la reportada por ILSA (2005) de 4,8 habitantes por cada hogar. También contrasta con las comunidades rurales a nivel nacional, donde la media de integrantes por hogar rural es de cinco, número de integrantes que representa el $78 \%$ de las familias (Álvarez y Restrepo, 2003). Asimismo, la cantidad de integrantes obtenida fue menor que en otro estudio realizado en el departamento de Antioquia (Colombia), donde la media fue de seis habitantes por cada hogar campesino (Álvarez y Restrepo, 2003). Sin embargo, la conformación de los hogares no fue homogénea considerando la repartición en grupos de las fincas. Los hogares del grupo 1 fueron los de menor número de integrantes.

El comportamiento de la media de integrantes de los grupos 2 y 3 fue similar entre sí y con la reportada para los hogares campesinos colombianos, donde se estimó una tendencia a disminuir de un promedio de 3,9 miembros por hogar en 2008, a 3,2 en 2020 (El Tiempo, 2015). Según el programa especial para la seguridad alimentaria en Centroamérica (FAO, 2006), Guatemala, Salvador, Honduras y Nicaragua tenían una cantidad mayor de personas en los hogares que la reportada en este estudio, pues sus valores variaron entre 5,8 y 5,4. El hecho de compartir estas características con países de Centroamérica permite entrever que las condiciones sociales de las familias rurales eran similares a las encontradas en las familias de este estudio. 
Cuadro 2. Indicadores sociales en tipos de hogares de cinco corregimientos la zona rural de Ibagué, Colombia. 2013.

Table 2. Social indicators in household types at five rural districts Ibague, Colombia. 2013.

\begin{tabular}{lccc}
\hline & $\begin{array}{c}\text { G1 } \\
\mathbf{n = 2 1}\end{array}$ & $\begin{array}{c}\text { G2 } \\
\mathbf{n = 2 8}\end{array}$ & $\begin{array}{c}\text { G3 } \\
\mathbf{n = 6}\end{array}$ \\
\hline Integrantes del hogar $\left(\mathrm{N}^{\circ}\right)$ & $3,00 \mathrm{a}$ & $4,25 \mathrm{ab}$ & $4,50 \mathrm{~b}$ \\
Hombres (\%) & 55,40 & 51,44 & 52,50 \\
Mujeres (\%) & 44,60 & 48,56 & 47,50 \\
Escolaridad del jefe de hogar $\left(\mathrm{N}^{\circ}\right)$ & $4,92 \mathrm{a}$ & $4,29 \mathrm{a}$ & $7,83 \mathrm{a}$ \\
Índice educativo & $1,20 \mathrm{a}$ & $2,43 \mathrm{ab}$ & $2,82 \mathrm{~b}$ \\
Índice de participación social & $1,31 \mathrm{a}$ & $1,46 \mathrm{ab}$ & $2,17 \mathrm{~b}$ \\
Índice de percepción. & $1,37 \mathrm{ab}$ & $1,12 \mathrm{a}$ & $1,73 \mathrm{~b}$ \\
\hline
\end{tabular}

G1: grupo 1/group 1; G2: grupo 2/group 2 y G3: grupo 3/group 3.

Los tres grupos mostraron una distribución porcentual por géneros similar, el porcentaje reportado de hombres fue de 55,4\% para el grupo 1, 51,44\% para el grupo 2 y $52,50 \%$ para el grupo 3 (Cuadro 2). Y la proporción de mujeres dentro de los hogares fue similar a la reportada por el Departamento Administrativo Nacional de Estadística (DANE) en el censo de 2005, según este, dentro de la población rural existía un 47\% de mujeres (CCJ, 2011). En otros países de América Latina la situación fue similar, por ejemplo, en el caso argentino las mujeres dentro del medio rural alcanzaron un $48,7 \%$, en Chile un $50,5 \%$ $y$ en Paraguay un 49,6\% (Chiappe, 2005).

La distribución de género evidenció porcentajes muy similares entre hombres (entre 51 y $55 \%$ ) y mujeres (entre 44 y 48\%). En términos de cantidad la distribución pudo ser similar, no es así en términos de derechos de igualdad, pues considerando las características machistas de la sociedad colombiana, que pudieron en muchos casos verse acentuadas en los campos, no permitió que las mujeres pudieran tener condiciones igualitarias, sobre todo en cuanto a oportunidades de estudio y laborales, así como a las actividades que debían realizar dentro del hogar.

El índice educativo (IE) de toda la muestra fue bajo ya que el $75 \%$ tuvo un IE menor o igual a 2,1, considerando esto como los grados que han sido cursados. Así, tres cuartas partes apenas habían alcanzado niveles educativos dentro de los primeros grados de primaria, en una población en la cual predominaban los adultos. Los hogares que del grupo 3 fueon los que mostraron un mayor índice educativo, siendo estas las fincas más grandes; seguidos por el grupo 2 (área intermedia) y por último, el grupo 1 que eran hogares que tenían los predios más pequeños.

Esto contrasta con lo encontrado en hogares campesinos del norte del Tolima, donde se reportaron los índices educativos más elevados en las fincas de tamaño medio $(2,9 \pm 1,2)$ y el índice más bajo en las fincas pequeñas y grandes $(1,6 \pm 1,1$ y $1,8 \pm 1,1$, respectivamente) (Rodríguez y Mora, 2014). Las fincas inmersas dentro de una economía cafetera mejor desarrollada como la detallada por Rodríguez y Mora (2014) permite mejores ingresos, que se ven reflejados en mayores índices educativos, en una relación que podría clasificarse como de doble vía, pues tener mejores ingresos permite acceder a una mejor educación por una parte y por otra, tener una mejor educación permite mejores niveles de ingresos.

Un estudio en hogares campesinos de México (CEPAL/FAO/RIMISP, 2003) dio a conocer que el jefe de hogar tenía de 2,9 años de estudio, sin embargo, en los otros dos grupos el indicador educativo fue menor. El nivel educativo de un hogar pudo sugerir una mejor disposición para el cambio y el logro de mejores condiciones de vida. Así, un hogar con más años de educación formal acumulados pudo estar más preparado para el ascenso en la escala social. Los países que hacen una mayor inversión en salud y educación han logrado índices mayores de desarrollo humano (PNUD, 2013).

La población menor de cinco años se reduce dentro de los tres grupos, tanto que el grupo 3 no tuvo individuos dentro de esta categoría. La categoría que contó con la mayor proporción de individuos fue la de 18-65 años, considerando estos como los individuos que se constituyen en fuerza laboral; seguido por las personas entre 5-18 años. En el caso particular del grupo 1, hubo una mayor proporción de individuos mayores de 65 años, que entre 5-18 años (Figura 3).

Según un estudio realizado en Antioquia, la distribución porcentual por edades de la población rural fue similar a la encontrada dentro del estudio, una mayor proporción de personas entre los 15 y los 64 años, y menor cantidad de individuos mayores de 65 años y menores de quince años (Álvarez et al., 


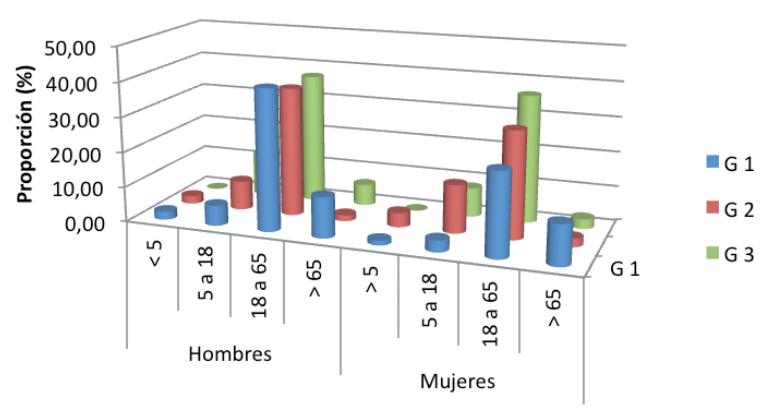

Figura 3. Distribución poblacional por edades y por géneros en cinco corregimientos del municipio de Ibagué, Colombia. 2013.

G1: grupo 1; G2: grupo 2; G3: grupo 3 .

Figure 3. Demographic distribution by ages and by genders in five townships of the Ibague municipality, Colombia. 2013.

G1: group 1; G2: group 2; G3: group 3 .

2007); por ende, los hogares en su mayoría estuvieron constituidos por individuos en edad laboral. Este resultado es importante considerarlo en conjunto con la cantidad de individuos reportados dentro de los hogares que tendió a ser alrededor de cuatro. Pues si se tiene en cuenta que existe una población mayor dentro del rango considerado como una edad laboral, se puede relacionar con uno de los fundamentos de la economía campesina, pues en esta se hace vital la inclusión de individuos de la misma familia como mano de obra dentro de las fincas.

Las medias de la edad del productor entre los diferentes grupos no mostraron diferencias significativas (Figura 3) entre sí y tuvieron valores de 47,67 para el grupo 2; 51,73 para el grupo 1 y 53,67 para el grupo 3. Estos datos concordaron con lo establecido por Fonseca (2002), ya que hizo referencia a que los jefes de hogar de las familias campesinas de Colombia sobrepasaron los 50 años. Una situación similar se presentó en un estudio realizado en la provincia de Neuquén en Argentina, donde el promedio de edad del jefe de hogar en un núcleo de familias campesinas fue de 61 años (Marín, 2010). La edad promedio de los jefes de hogar del presente estudio fue menor que la reportada por estudios previos en los países de Centroamérica, pues dicha media fue de 45 años (FAO, 2006).

\section{Usos del suelo}

En la Cuadro 3 se puede apreciar el área media de los grupos de fincas y la relación entre usos agrícolas y usos pecuarios, para establecer una diferencia numérica entre las tendencias dominantes de cada grupo. El área total hace referencia a los datos de área brindados por los productores, mientras que el área calculada es la suma de las áreas reportadas por los productores de cada uno de los usos de suelo y fue calculada por los investigadores.

El área cultivada de los grupos 1 y 2 , con 1,17 y 2,59 ha/finca, respectivamente, no mostraron diferencias significativas $(p>0,05)$ entre sí, mientras que el grupo 3 con una media de 4,77 si fue significativamente diferente. Estos resultados fueron concordantes con los reportados por la Federación Nacional de Cafeteros (2005) que indicó que el 89\% de las fincas cafeteras tenían un área cultivada de tres hectáreas. Otros sistemas agroforestales, como los cacaotales en Colombia, se han caracterizado porque

Cuadro 3. Medias del área total de la finca, área estimada y relación de área entre agricultura y ganadería en cinco corregimientos del municipio de Ibagué, Colombia. 2013.

Table 3. Averages of the total area of the farms, estimated area and ratio between the agricultural and the livestock area in five townships of Ibague municipality, Colombia. 2013.

\begin{tabular}{lccc}
\hline & G1 & G2 & G3 \\
\hline Área total & $8,95 \pm 15,60 \mathrm{~b}$ & $4,74 \pm 3,55 \mathrm{~b}$ & $59,68 \pm 112,23 \mathrm{a}$ \\
Área calculada & $3,71 \pm 5,88 \mathrm{a}$ & $3,15 \pm 2,31 \mathrm{a}$ & $52,43 \pm 111,51 \mathrm{~b}$ \\
Agricultura/ganadería & $0,65 \pm 0,49$ & $1,79 \pm 4,24$ & $1,68 \pm 2,13$ \\
\hline
\end{tabular}

G1: grupo 1/group 1; G2: grupo 2/group 2 y G3: grupo 3/group 3. 
el $92 \%$ de las fincas tienen entre una y cincuenta hectáreas, concentrándose la mayoría de las fincas en un rango entre cinco y diez hectáreas, por lo tanto, estas fincas tienen una superficie mayor que las fincas del presente estudio. No obstante, no todas las fincas cacaoteras colombianas puedan enmarcarse dentro de la economía campesina, pues existe en este cultivo un porcentaje de fincas industrializadas con un área mayor (Espinal et al., 2005).

Las fincas del grupo 1 poseían una importante proporción del suelo bajo pasturas, a pesar de ser los predios más pequeños con una superficie promedio de 3,71 $\pm 5,88$ ha. Fueron predios típicos de economía campesina, en los cuales se combinaban actividades agrícolas y pecuarias y estaban distribuidos geográficamente en los diferentes pisos térmicos de la zona de estudio, si bien también cultivaban café, lo hacían en menor medida y por tanto, los cultivos de musáceas, otros productos como frutales, yuca y cacao, se veían en una mayor proporción. El grupo 2 estaba conformado básicamente por predios dedicados al cultivo del café; el área en pasturas de estas fincas era bastante reducida, pero también manejaban animales, además cultivaban plátano, banano y cacao. Fueron las fincas que geográficamente estaban distribuidas básicamente en la franja apropiada para el cultivo del café, entre los 1200 y 1800 m (Figura 4), y como es característico de las fincas cafeteras campesinas su superficie era pequeña $(3,15 \pm 2,31 \mathrm{ha})$.

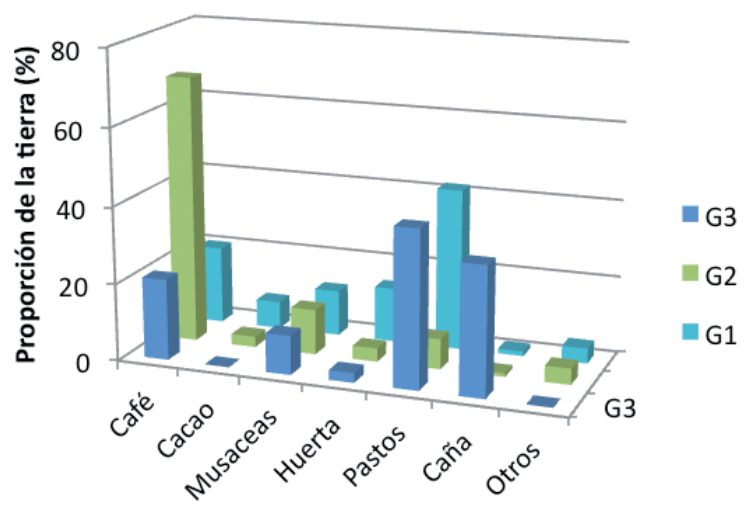

Figura 4. Usos del suelo en diferentes tipos de fincas en la zona rural de Ibagué, Colombia, 2013.

G1: grupo 1; G2: grupo 2; G3: Grupo 3.

Figure 4. Land uses in different farms types in Ibague rural area, Colombia, 2013.

G1: group 1; G2: group 2; G3: group 3 .
En las fincas del grupo 3 predominaban las pasturas y los cultivos de caña de azúcar y básicamente se dedicaban a la ganadería. Fueron fincas con características poco comunes, particularmente por su gran extensión y esto hacía que quedaran por fuera del prototipo de las fincas encontradas en la zona de estudio $(52,43 \pm 111,51$ ha). Además de la ganadería, cultivaban una pequeña área de café y plátano. Forero (2003) hace una descripción de finca campesina similar a las de este estudio, pues hizo referencia a cultivos de café, plátano, frutales, yuca, maíz y caña de azúcar; sin embargo, hizo referencia a cultivos que no fueron descritos en este estudio como la papa, el arroz y el ajonjolí, principalmente. En dicho estudio también se mencionó la producción de carne y leche, que también se observa en el municipio de Ibagué, razón por la cual existe un área importante dispuesta a las pasturas (Forero, 2003). Cultivos tales como el arroz y el ajonjolí se han asociado a fincas de mayor tamaño y mayor inversión tecnológica, por lo cual es probable que no hayan sido reportadas en este estudio. En cuanto a la papa, este es un cultivo de tierras altas, y las fincas ubicadas en esta zona para este estudio dedicaban su superficie a la ganadería.

Las fincas del grupo 1 se ubicaron en tierras más bajas, aptas para la ganadería; mientras que las fincas del grupo 2 a altitudes donde es posible el cultivo del café, y las fincas del grupo 3 no tuvieron un rango de altitud definido, puesto que sus actividades no se enfocaban a una sola actividad (Figura 5).

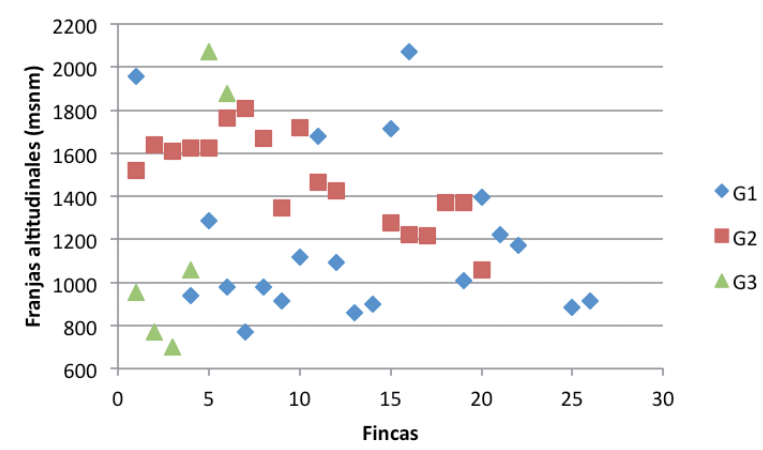

Figura 5. Distribución de las fincas analizadas en diferentes franjas altitudinales de la zona rural de Ibagué, Colombia. 2013

G1: grupo 1; G2: grupo 2; G3: Grupo 3.

Figure 5. Distribution of farms analyzed in different altitudinal bands rural Ibague, Colombia. 2013. G1: group 1; G2: group 2; G3: group 3 . 


\section{Capacidad instalada}

Los grupos 1 y 3 contaron con una capacidad instalada mayor y significativamente diferente a las fincas del grupo 2. Eso se expresó en un índice promedio mayor para estos dos grupos de fincas $(3,08$ y 3,67, respectivamente) respecto al grupo $2(1,38)$ (Cuadro 4). Sin embargo, en los tres casos la infraestructura fue incipiente y muy rudimentaria. Camargo y Espinosa (2011) reportaron que en el municipio de Tuta, Boyacá se observó una situación similar en la cual las familias contaban con una vivienda, pero no con recursos para mejorar las instalaciones necesarias para la producción pecuaria. Considerando que las fincas del grupo 3 tenían mayores ingresos, se deduce que esta característica estuvo relacionada en gran medida con el desarrollo tecnológico que se encuentre en las fincas.

Cuadro 4. Capacidad instalada de las fincas analizadas en la zona rural de Ibagué, Colombia. 2013.

Table 4. Installed capacity of the analyzed farms in Ibague rural area, Colombia. 2013.

\begin{tabular}{lccc}
\hline Instalación & G1 & G2 & G3 \\
\hline Invernaderos agrícolas & $0 \%$ & $4,16 \%$ & $16,67 \%$ \\
Corrales & $34,62 \%$ & $8,33 \%$ & $66,67 \%$ \\
Porquerizas & $19,2 \%$ & $8,33 \%$ & $16,7 \%$ \\
Galpones & $30,27 \%$ & $0 \%$ & $0 \%$ \\
Estanques piscícolas & $26,92 \%$ & $0 \%$ & $16,67 \%$ \\
Bodega & $19,2 \%$ & $4,17 \%$ & $50 \%$ \\
Cercas & $92 \%$ & $75 \%$ & $100 \%$ \\
\hline
\end{tabular}

G1: grupo 1/group 1; G2: grupo 2/group 2 y G3: grupo 3/group 3 .

\section{Artefactos y equipos}

El grupo 3 con una media de 3,17, fue significativamente diferente de los otros dos grupos con medias de 1,88 para el grupo 1 y 1,96 para el grupo 2 (Cuadro 5). Según estos resultados aún en el grupo 3 fueron muy básicos los bienes de producción presentes en las fincas, por lo tanto, los productores se han visto obligados a trabajar de una manera
Cuadro 5. Porcentajes de posesión de artefactos y equipos encontrados en las fincas analizadas en la zona rural de Ibagué, Colombia. 2013.

Table 5. Artefacts possession by percentage and equipment founded in the analyzed farms in Ibague rural area, Colombia. 2013.

\begin{tabular}{lccc}
\hline Instalación & G1 & G2 & G3 \\
\hline Sistemas de riego & $0 \%$ & $0 \%$ & $17 \%$ \\
Vehículo & $23,1 \%$ & $8,33 \%$ & $50 \%$ \\
Bombas de espalda & $84,62 \%$ & $87,5 \%$ & $83,33 \%$ \\
Bomba de agua & $11,5 \%$ & $4,17 \%$ & $0 \%$ \\
Picadora de pastos & $27 \%$ & $0 \%$ & $50 \%$ \\
Motosierra & $15 \%$ & $0 \%$ & $67 \%$ \\
Generador eléctrico & $0 \%$ & $0 \%$ & $33,33 \%$ \\
Despulpadora & $23,08 \%$ & $75 \%$ & $16,67 \%$ \\
\hline
\end{tabular}

G1: grupo 1/group 1; G2: grupo 2/group 2 y G3: grupo 3/group 3.

tradicional con herramientas como el machete y el azadón. Esta situación fue similar a la reportada por Camargo y Espinosa (2011), donde en un estudio realizado en Tuta (Boyacá), se reportaron dos tipos de fincas, las fincas tradicionales donde los bienes de producción eran herramientas de uso tradicional como la pica, el azadón, la hoyadora y el machete, y otras fincas que eran similares a las reportadas en el grupo 3 del presente estudio que contaban además de las herramientas tradicionales, con motobombas y en algunos casos sistemas de riego.

\section{Capital financiero}

Respecto a los ingresos agrícolas los grupos 1 y 2 fueron iguales entre sí, pero estadísticamente diferentes del grupo 3. Esta variable mostró una diferencia importante dentro de los grupos, pues determinó un mayor ingreso por conceptos agrícolas para el grupo 3 (Cuadro 6). La media de ingresos del grupo 3 fue similar a los ingresos reportados por Forero (2010) para productores hortofrutícolas de Fómeque y Piedecuesta en Santander, con ingresos anuales de 12545000 . Mientras que los grupos 1 y 2 reportaron ingresos similares a los minifundistas de Nariño con ingresos agrícolas de 1316000 anuales. 
Cuadro 6. Media de ingresos por hectárea en las fincas analizadas en la zona rural de Ibagué, Colombia. 2013.

Table 6. Average of incomes by hectare in the analyzed farms in the rural area of Ibague, Colombia. 2013.

\begin{tabular}{|c|c|c|}
\hline \multirow{8}{*}{ 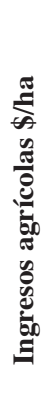 } & \multicolumn{2}{|c|}{ G1 } \\
\hline & Media & D.E. \\
\hline & $723122 a^{*}$ & 1967344 \\
\hline & \multicolumn{2}{|c|}{ G2 } \\
\hline & Media & D.E \\
\hline & $1088861 \mathrm{a}^{*}$ & 746846 \\
\hline & \multicolumn{2}{|c|}{ G3 } \\
\hline & Media & D.E \\
\hline & $1721732 b^{*}$ & 2052636 \\
\hline
\end{tabular}

* $\mathrm{P}<0,05$; medias con letra diferente muestran diferencias significativas / $\mathrm{P}<0,05$; means with different letter show significant differences.

G1: grupo 1/group 1; G2: grupo 2/group 2 y G3: grupo 3/group 3.

\section{AGRADECIMIENTOS}

Los autores desean agradecer al Comité Central de Investigaciones de la Universidad del Tolima por el financiamiento dado al proyecto "Análisis socioeconómico y ambiental de productores agropecuarios en tres zonas estratégicas del departamento del Tolima". Un agradecimiento especial a los productores de la zona rural de Ibagué que participaron en el estudio.

\section{LITERATURA CITADA}

Alcaldía de Ibagué. 2010. Ibagué en cifras 2008-2009. http://www.tolima.gov.co/municipios/muni/ibague (consultado 24 ene. 2014).

Álvarez, M., y L.F. Restrepo. 2003. La variedad de alimentos disponibles en el hogar: Metodología para identificar vulnerabilidad a la inseguridad alimentaria y nutricional en hogares campesinos. RESPYN. 4 (4). http://www.respyn.uanl.mx/iv/4/articulos/meto-seg. htm. (consultado 24 abr. 2014).

Álvarez, M.C., L.P. Mancilla, y J.E. Cortés. 2007. Caracterización socioeconómica y seguridad alimentaria de los hogares productores de alimentos para el autoconsumo, Antioquia-Colombia. Agroalimentaria 12(25):112-113

Balzarini M.G., L. Gonz'Alez, M. Cuadroda, F. Casanoves, J.A. Di Rienzo, y C.W. Robledo. 2008. InfoStat, versión 2008. Grupo InfoStat, FCA, Editorial Brujas, Córdoba, ARG.

Berdegué, J., y B. Larrain. 1988. Cómo trabajan los campesinos. Editorial Celater, COL.

Bermúdez M.B., J. Mora-Delgado, y M. Gómez. 2011. Tipología de sistemas de producción en la ecorregión cafetera del Tolima. En: J. Mora-Delgado, y V. Holguín, editores, Medios de vida y materiales orgánicos en fincas campesinas (métodos de análisis en fincas de la ecorregión cafetera). Universidad del Tolima-Red Alma Mater, Ibagué, COL. p. 57-66.

Bolaños, E. 2012. Muestra y muestreo. Asignatura: Estadística para el Desarrollo Tecnológico. Universidad Autónoma del Estado de Hidalgo, México. http://www.uaeh.edu.mx/docencia/P_Presentaciones/ tizayuca/gestion_tecnologica/muestraMuestreo.pdf (consultado 12 jul. 2015).

Camargo, R., y M. Espinosa. 2011. Medios de vida en familias campesinas del municipio de Tuta, Boyacá. Cultura Científica 9:54-61.

CCJ (Comisión Colombiana de Juristas). 2011. La política agraria y los derechos de las mujeres en Colombia. CCJ, COL.

CEPAL/FAO/RIMISP. 2003. La pobreza rural en América Latina: lecciones para una reorientación de las políticas. Seminarios y Conferencias No. 27. CEPAL, Santiago de Chile, CHI.

Chiappe, M. 2005. La situación de las mujeres rurales en la agricultura familiar de cinco países de América Latina. https://www.google.com.co/url?sa=t\&rct=j\&q=\&esrc $=$ s $\&$ source $=$ web $\& \mathrm{~cd}=4 \& \mathrm{cad}=\mathrm{rja} \& \mathrm{ved}=0 \mathrm{CD} \mathrm{sQFjA}$ D\&url=http $\% 3 \mathrm{~A} \% 2 \mathrm{~F} \% 2 \mathrm{Fwww}$.generoycomercio. org\%2Fareas\%2Finvestigacion\%2FMujeresrurales. doc\&ei=LAXsUozeJob (consultado 12 dic. 2013).

Di Rienzo, J.A., F. Casanoves, M.G. Balzarini, L. González, M. Cuadroda, y C.W. Robledo. 2008. InfoStat, versión 2008. Grupo InfoStat, FCA, Universidad Nacional de Córdoba, ARG.

El Tiempo. 2015. Tamaño de los hogares colombianos se reducirá más que en el resto de América Latina. http://www.eltiempo.com/archivo/documento/CMS6607687 (consultado 13 may. 2015).

Espinal, C.F., H.J. Martínez, L. Ortiz. 2005. La cadena del cacao en Colombia; una mirada global de su estructura y dinámica. http://www.agronet.gov.co/www/docs_ 
agronet/2005112145659_caracterizacion_cacao.pdf (consultado 24 ene. 2014).

FAO. 2006. Programa especial para la seguridad alimentaria. http://www.pesacentroamerica.org/biblioteca/ pequenos_productores/capitulo2.pdf (consultado 17 dic. 2013).

Federación Nacional de Cafeteros de Colombia. 2005. Extensión rural en la Federación Nacional de Cafeteros de Colombia. http://www.corpoica.org.co/ sitioweb/Archivos/Foros/ExtensinFederacinCafeteros. pdf (consultado 3 feb. 2014).

Fonseca, A. 2002. Colombia: escenario social económico e institucional de la actual crisis cafetera. http://www. cepal.org/ddpe/noticias/noticias/9/9719/cafecolombia. pdf (consultado 17 dic. 2013).

Forero, J. 2003. Economía campesina y sistema alimentario en Colombia: aportes para la discusión sobre seguridad alimentaria. http:// www.javeriana.edu.co/ear/d_des_rur/documents/ campesinadoysistemaalimentarioencolombia.pdf (consultado 25 ene. 2014).

Forero, J. 2010. Economía campesina, pobreza, tierra y desplazamiento en Colombia. https://www. google.com.co/url?sa=t\&rct=j\&q=\&esrc=s\& source $=$ web $\& c d=4 \& c$ ad $=$ rja $\&$ ved $=0 C D g Q F j$ AD\&url=http $\% 3 \mathrm{~A} \% 2 \mathrm{~F} \% 2 \mathrm{Fww}$.planetapaz. org $\% 2$ Findex.php $\% 2$ Fbiblioteca6\%2Fdocumentosrelacionados $\% 2 \mathrm{Fdoc} \_d o w n l o a d \% 2 \mathrm{~F} 6$-economiascampesinas-pobreza-tierra-y-desplazamientoen-colombia\&ei=NivoUr_GGLOksQTa6YD QCw\&usg=AFQjCNF3d6uO9igAT6qAWYZDAQB mmjLhA\&bvm =bv.60157871, d.eW 0 (consultado 24 ene. 2014).

García, C., y L. Calle. 1998. Consideraciones metodológicas para la tipificación de sistemas de producción bovina a partir de fuentes secundarias. Corpoica Cienc. Tecnol. Agropecu. 2:6-15.

Gobernación del Tolima. 2011. Ibagué en cifras 2000-2010. http://www.tolima.gov.co/municipios/muni/ibague/ (consultado 3 feb. 2014).

Guio, A., J. Muñoz, H.J. Andrade, M. Segura, S. Lozada, J. Mora-Delgado, M.J. Gómez, y M. Barrios. 2014. Diseño del esquema de PSA para la cuenca alta del río Combeima, municipio de Ibagué (Tolima, Colombia). Convenio de Cooperación $\mathrm{N}^{\circ}$ 022. Universidad del Tolima-Cortolima-Patrimonio Natural, Ibague, COL.
Hernández-Sampieri, R., F. Collado, y P. Baptista. 2006. Metodología de la investigación. McGrawHill, MEX.

ILSA (Instituto Latinoamericano para una Sociedad y un Derecho Alternativos). 2005. Impactos del TLC con Estados Unidos sobre la economía campesina en Colombia. http://ilsa.org.co:81/biblioteca/dwnlds/ otras/tlc/cap3.pdf (consultado 3 feb. 2014).

Landais, E. 1998. Modelling farm diversity new approaches to typology building in France. Agric. Sys. 58:505527.

Marín, H.G. 2010. Estrategias campesinas de vida de las familias rurales de Quili Malal. En: Asociación latinoamericana de sociología, editor, Memorias del VII Congreso Latinoamericano de Sociología Rural. Porto Galinhas, CHI. p. 11.

Mora, J., J.C. Calderón, y S.M. Gómez. 2011. El componente pecuario en fincas campesinas de la ecorregión cafetera del departamento del Tolima (Colombia). Luna Azul 32:16-31.

Murray, R. S., y L.J. Stephens. 2009. Estadística. $4^{\text {ta }}$ edición. McGraw-Hill, MEX.

PNUD (Programa de Naciones Unidas para el Desarrollo). 2013. Informe sobre desarrollo humano 2013, El ascenso del sur: progreso humano en un mundo diverso. PNUD, N.Y, USA.

Rodríguez, P.A., y J.R. Mora. 2014. La diversificación en sistemas agroforestales cafeteros del Tolima: una respuesta a la crisis. Agrofor. Neotrop. 4:28-32.

Rodríguez, P.A., y J.R. Mora. 2015. Capital social en la cuenca hidrográfica del río Combeima y su papel en la gestión del recurso hídrico. Anteproyecto de Tesis $\mathrm{PhD}$, Universidad del Tolima, Ibagué, COL.

Stiles, F.G. 1998. Especies de aves endémicas y casi endémicas de Colombia. En: M.E. Chaves, y N. Arango, editores, Informe Nacional sobre el estado de la biodiversidad 1998-Colombia. Instituto Alexander von Humboldt, PNUMA, Ministerio del Medio Ambiente, Santa Fé de Bogotá, COL. p. 378-432.

Vanegas, M. 2002. Estudio complementario del caso Ibagué. Convenio IDRC - OPS/HEP/CEPIS Ibagué. http:// www.bvsde.paho.org/bvsaar/e/proyecto/complemen/ casos/ibague.pdf. (consultado 24 abr. 2014). 